\title{
Comparative assessment of qualitative and quantitative perfusion with dual-energy CT and planar and SPECT-CT V/Q scanning in patients with chronic thromboembolic pulmonary hypertension
}

\author{
Rahul D. Renapurkar ${ }^{1}$, Michael A. Bolen ${ }^{1,2}$, Sankaran Shrikanthan ${ }^{1}$, Jennifer Bullen ${ }^{3}$, Wadih Karim ${ }^{1}$, \\ Andrew Primak ${ }^{4}$, Gustavo A. Heresi ${ }^{5}$ \\ ${ }^{1}$ Imaging Institute, ${ }^{2}$ Heart and Vascular Institute, ${ }^{3}$ Quantitative Health Sciences, Cleveland Clinic, Cleveland, OH, USA; ${ }^{4}$ Siemens Medical Solutions, \\ Malvern, PA, USA; ${ }^{5}$ Department of Pulmonary and Critical Care Medicine, Respiratory Institute, Cleveland Clinic, Cleveland, OH, USA \\ Contributions: (I) Conception and design: RD Renapurkar, J Bullen; (II) Administrative support: All authors; (III) Provision of study materials \\ or patients: RD Renapurkar, S Shrikanthan, MA Bolen, GA Heresi; (IV) Collection and assembly of data: All authors; (V) Data analysis and \\ interpretation: All authors; (VI) Manuscript writing: All authors; (VII) Final approval of manuscript: All authors. \\ Correspondence to: Rahul D. Renapurkar, MD. Thoracic Imaging, L10, Imaging Institute, Cleveland Clinic, Cleveland, OH 44195, USA. \\ Email: renapur@ccf.org.
}

Background: The purpose of this study was to compare the qualitative and quantitative assessment of perfusion on dual-energy CT (DECT) and planar and single photon emission computed tomography (SPECT)-CT V/Q scanning in patients with chronic thromboembolic pulmonary hypertension (CTEPH).

Methods: Nineteen patients with known CTEPH underwent both DECT and SPECT-CT V/Q scanning. Sixteen of these patients underwent planar V/Q imaging concurrently. Two readers independently graded DECT-perfused blood volume (PBV) defects on a four-point scale $(0=$ normal, $1=$ mild $<25 \%, 2=$ moderate $25-50 \%, 3=$ severe $>50 \%$ ). A grade was given for each lung lobe and for each of 18 lung segments. One reader graded the SPECT-CT images similarly. Quantitative measurements of lung perfusion were calculated with DECT and planar V/Q scanning for 16 of these patients.

Results: The inter-reader agreement on DECT was strong with agreement in $85 \%$ (258/304) of segments $($ kappa $=0.86)$ and $84 \%(80 / 95)$ of lobes (kappa $=0.82)$. The inter-modality agreement between DECT and SPECT-CT was lower. Readers 1 and 3 agreed in only 34\% (103/304) of segments (kappa =0.25) and $33 \%(31 / 94)$ of lobes (kappa =0.22). Agreement between readers 2 and 3 was similar. Correlation between quantitative measurements with DECT and planar V/Q imaging was poor and ranged from 0.01 to 0.45.

Conclusions: Inter-observer agreement in subjective grading of PBV maps is excellent. However, intermodality agreement between DECT and SPECT-CT is modest. Automated quantification values of PBV maps correlate poorly with established tools like planar V/Q imaging. These differences need to be kept in mind during clinical decision making.

Keywords: Dual-energy CT (DECT); single photon emission computed tomography-CT V/Q scanning (SPECT-CT V/Q scanning); chronic thromboembolic pulmonary hypertension (CTEPH)

Submitted Jan 17, 2018. Accepted for publication Mar 16, 2018.

doi: $10.21037 /$ cdt.2018.05.07

View this article at: http://dx.doi.org/10.21037/cdt.2018.05.07 


\section{Introduction}

Chronic thromboembolic pulmonary hypertension (CTEPH) is one of the increasingly recognized causes of pulmonary hypertension $(\mathrm{PH})$. The diagnosis is based on establishing proximal and/or distal thrombotic disease as a cause of $\mathrm{PH}$ [mean pulmonary arterial pressure (mPAP) $\geq 25 \mathrm{mmHg}$ and pulmonary capillary wedge pressure is $\leq 15 \mathrm{mmHg}$ ] (1). Patients with CTEPH often present with nonspecific symptoms such as dyspnea and the diagnosis can be challenging. Often, the diagnosis is suggested by echocardiography that demonstrates elevated right ventricular systolic pressure (RVSP). Once the diagnosis of $\mathrm{PH}$ is established, CTEPH should be considered in the differential diagnosis even in patients without a history of venous thromboembolism or risk factors for thromboembolism (2). Surgery is the definitive treatment of CTEPH, with pulmonary thromboendarterectomy (PTE) offering excellent short-term and long-term outcomes (3-5). To establish chronic thromboembolic disease (CTED) as a cause of $\mathrm{PH}$, several noninvasive imaging tests are available. Ventilation-perfusion (V/Q) scanning is generally accepted as the initial screening test of choice due to its ease of availability and interpretation, high sensitivity and excellent negative predictive value (6). V/Q scanning is more sensitive than computed tomography pulmonary angiography (CTPA) for diagnosis of CTEPH, and a negative V/Q scan effectively rules out CTEPH (7). However, V/Q scanning is not free from drawbacks and some of the major limitations of planar V/Q scanning include the high number of non-diagnostic scans and false positives. Thus, once the diagnosis of CTEPH is suggested by V/Q scanning, an additional cross-sectional imaging such as CTPA is imperative to confirm the diagnosis and define the anatomic extent of disease.

Newer techniques such as single photon emission computed tomography (SPECT) V/Q scanning and dual energy computed tomography (DECT) have emerged in the past decade. SPECT V/Q scanning in combination with low dose CT for attenuation correction (SPECTCT V/Q scanning) overcomes some of the limitations of planar 2-D imaging and increases accuracy of mapping of perfusion defects (PD) in the lung. Several studies have shown the superiority of SPECT V/Q imaging over planar techniques in the assessment of pulmonary thromboembolic disease (8). Parallel to the evolution of SPECT imaging has been the emergence of DECT. DECT allows a base material decomposition and quantification of materials such as iodine within a voxel of tissue (9). Quantification of amount of iodine within a voxel allows generation of perfused blood volume (PBV) images, which have proven to be a good surrogate marker of lung perfusion (10).

In evaluation of CTEPH patients, imaging plays an important role beyond its diagnosis. Some of the critical factors to evaluate preoperatively are the anatomic distribution and extent of disease, assessment of pulmonary vascular resistance (PVR), and assessment of lung perfusion and microvasculature. DECT allows automated quantification of $\mathrm{PBV}$ maps, which might serve as an objective tool for assessment of lung perfusion (11). Small studies comparing PBV maps to planar and SPECT V/Q imaging have shown to modest to good correlation $(10,12,13)$. However, data on this topic is still lacking and some of the critical questions that remain unclear are how to use the PBV maps for subjective assessment, as well as the potential use of automated quantification values in preoperative planning.

In this study, we sought to assess inter-reader agreement in subjective grading of perfusion on PBV images. Additionally, we compared the concordance of subjective grading of perfusion on DECT and SPECT-CT V/Q scanning. Finally, we compared the quantification of PBV maps with those obtained by planar V/Q scanning and assessed for their correlation.

\section{Methods}

\section{Study population}

This retrospective study was performed with local institutional review board approval and a waiver of individual informed consent. Over a period of 18 months (January 2014 to June 2015), 19 patients with known CTEPH underwent both DECT and SPECT-CT V/ Q scanning. The diagnosis of CTEPH was made using clinical presentation and imaging tests. Sixteen of these patients also underwent planar V/Q imaging concurrently during the SPECT acquisition. Mean time interval between DECT and SPECT-CT V/Q scanning was 15 days. Eight of these patients were females and 11 were males. Average age of the patient was 53.7 years. Exclusion criteria included patients with contraindications to contrast administration such as poor renal function and contrast dye allergies. Pregnant females were also excluded from the study. Patients with body weight greater than 400 pounds (lbs) were excluded from the study as the image noise was felt to 
Table 1 Demographic information of the study population

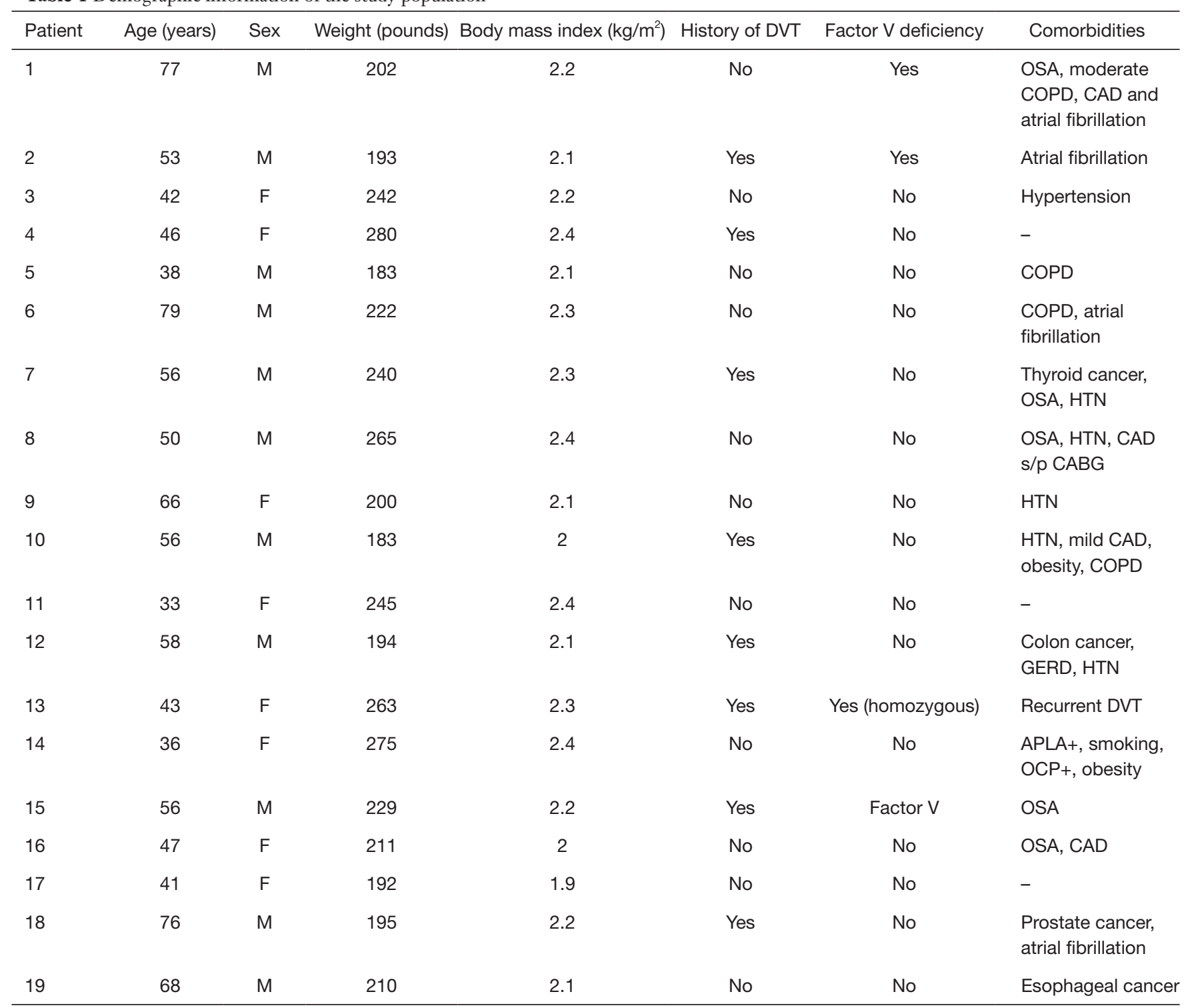

DVT, deep vein thrombosis; OSA, obstructive sleep apnea; COPD, chronic obstructive pulmonary disease; HTN, hypertension; CAD, coronary artery disease; CAD, coronary artery disease; CABG, coronary artery bypass surgery; GERD, gastroesophageal reflux disease; APLA, antiphospholipid antibody; OCP, oral contraceptive pills; M, male; F, female.

be unacceptable. Body weights ranged from 183 to $280 \mathrm{lbs}$, with an average of $222.4 \mathrm{lbs}$. Detailed demographic data is listed in Table 1.

\section{CT protocol}

All CT scans were performed on a Somatom Definition Flash CT scanner (Siemens Healthcare, Forchheim, Germany). Default peak kilovoltage $(\mathrm{kVp})$ settings for the standard DECT-PE protocol were 100 and $140 \mathrm{Sn} \mathrm{kVp}$, with an additional $80 / 140 \mathrm{Sn} \mathrm{kVp}$ pair available. The other scanning parameters for the standard DECT-PE protocol were as follows: quality reference mAs of 170 for $100 \mathrm{kVp}$ and 145 for $140 \mathrm{Sn} \mathrm{kVp}, 64 \mathrm{~mm} \times 0.6 \mathrm{~mm}$ collimation with z-flying focal spot, $0.28 \mathrm{~s}$ rotation time, and pitch of 0.55 . Amount of contrast bolus was calculated by using weight based P3T software (MedRad, Bayer Healthcare, Germany) with an injection rate of $5 \mathrm{~mL} / \mathrm{s}$. Bolus tracking was used 
with region of interest (ROI) on the main pulmonary artery. A caudocranial scan direction was used. All patients were scanned by a dedicated technologist who was trained to run the DECT-PE protocol described above. Radiation dose in terms of volume CT dose index (CTDIvol) and dose length product (DLP) were recorded for each DECT scan and the effective dose from DECT was obtained by multiplying the average DLP value over the entire cohort by the adult chest $\mathrm{k}$-factor of $0.014 \mathrm{mSv}^{*} \mathrm{mGy}^{-1 *} \mathrm{~cm}^{-1}$.

\section{Planar and SPECT-CT V/Q scanning protocol}

Ventilation images were obtained first using 99mTc-labeled diethylene triamine pentaacetic acid (99mTc-DTPA) aerosol followed by perfusion images after intravenous injection of 99mTc-labeled macroaggregated albumin (99mTc-MAA). Planar images were obtained in multiple views including anterior, posterior, right and left oblique and both lateral views using a dual headed camera.

SPECT/CT images were obtained using a Siemens Symbia T6 scanner (Siemens Healthcare, Forchheim, Germany). Images were acquired centering the lungs in the field-of-view. The $99 \mathrm{mTc}$ window was centered at 140 kilo-electron volt ( $\mathrm{keV})$ with $15 \%$ width (range, 129.5-150.5 keV). SPECT data were acquired in a step-and-shoot sequence with a noncircular orbit (dual detectors in $180^{\circ}$ configuration; low-energy high-resolution collimators). After completion of SPECT, attenuation correction/localization non-contrast CT was acquired [effective milliampere-second (mAs): $30, \mathrm{kV}: 130$, slice: $5.0 \mathrm{~mm}$ Acq $6 \mathrm{~mm} \times 2.0 \mathrm{~mm}$, rotation time: $1.0 \mathrm{sec}$, Scan: Craniocaudal, Pitch 1.0].

\section{Data reconstruction}

The linearly mixed DE images that serve as the surrogate for the conventional $120 \mathrm{kV} \mathrm{SE}$ images were reconstructed at 1-mm thickness using medium-soft convolution kernel (B31f) for pulmonary vasculature and sharp convolution kernel (B70f) for lungs. The high- and low-kV images used for $\mathrm{DE}$ post-processing were reconstructed at $1-\mathrm{mm}$ using the DE-specific medium smooth kernel with iodine beam hardening correction (D33f). The iodine (PBV) maps were generated using the syngo.via DE Lung PBV software (version VA20). The principle of PBV calculation involves loading both $80 / 100-\mathrm{kV}$ and $140-\mathrm{kV}$ images into the software and calculating the iodine content of each voxel through a three-material-decomposition algorithm for air, soft tissue, and iodine. The lung parenchyma is color coded with 16-bit color coding. PBV values are calculated automatically in $\mathrm{HU}$ based on the enhancement patterns in both lungs. The images were viewed on Picture Archiving and Communication System (PACS) (Agfa) using standard mediastinal (window width, $400 \mathrm{HU}$, window center, $40 \mathrm{HU}$ ) and lung parenchymal (window width, 1,600 HU, window center, $-600 \mathrm{HU}$ ) settings

SPECT images were reconstructed on the scanner console workstation using iterative reconstruction. Both non-attenuation and CT attenuation-corrected images were reconstructed using FLASH3D software with 6 iterations, 10 subsets and 8.40 Gaussian filter.

\section{Image analysis}

The primary aim of this study was to assess the interreader variability in the subjective grading of $\mathrm{PD}$ on dual energy CT and to compare the grading of subjective PD using DECT-PBV maps and SPECT-CT V/Q scanning. Secondary aim of the study was to compare the automated quantification of the perfusion using DECT to quantitative perfusion generated by planar V/Q scanning. Only the perfusion information (from V/Q scanning) was used for analysis.

Two readers (reader 1 and 2 with 8 and 6 years of experience in cardiothoracic imaging respectively) independently graded PBV defects in 19 patients on a fourpoint scale $(0=$ normal, $1=$ mild $<25 \%, 2=$ moderate $25-50 \%$, $3=$ severe $>50 \%)$. For each patient, a grade was given for the five lung lobes, as well as for each of 18 segments within the lungs. One reader (reader 3 with 10 years of experience in nuclear medicine) analyzed the SPECT-CT images and graded those on a similar four-point scale. Segments that were affected by artifacts (such as contrast/tracer related artifacts and motion) were graded as non-gradable. Quantitative measurements of lung perfusion were collected with both DECT and planar V/Q scanning for 16 of the patients.

\section{Statistical methods}

Agreement was assessed using percent agreement and Cohen's kappa at the segment, lobe, and patient level (using the four-point scale discussed above). Linear weights were used for Cohen's kappa. For the assessment of agreement at the patient level, standard asymptotic confidence intervals for the percent agreement and kappa were constructed. For 


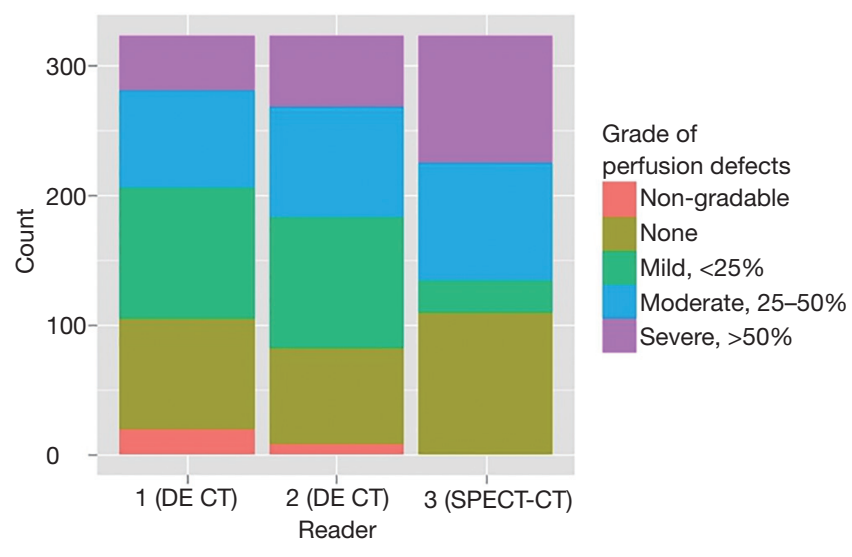

Figure 1 Distribution of segment-level grading of perfusion defects by reader (19 patients, 323 segments).

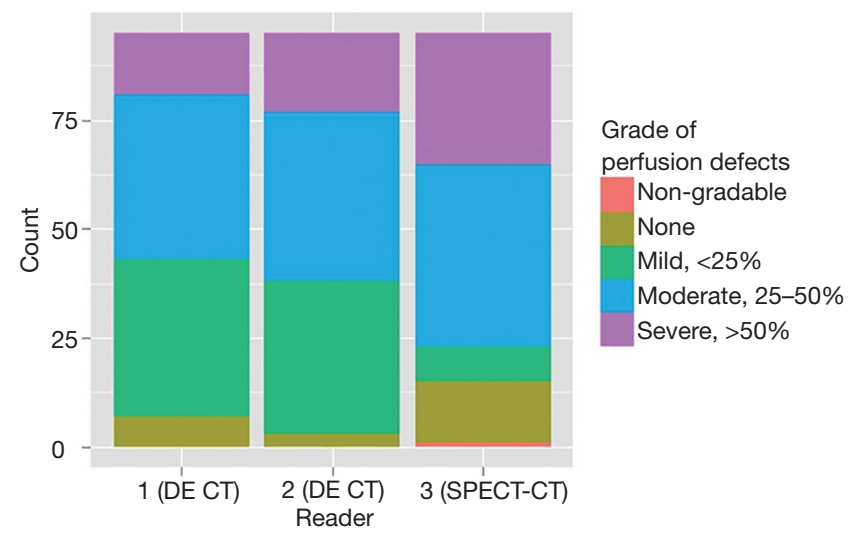

Figure 2 Distribution of lobe-level grading of perfusion defects by reader (19 patients, 95 lobes).

the assessments at the lobe- and segment-level, percentile bootstrap confidence intervals were computed for the percent agreement and kappa, where 100,000 bootstrap samples were generated (each sampled with replacement at the patient level). This approach was used to account for the clustering of observations within a patient (five lobes and 17 segments per patient).

The inter-reader agreement on DECT was assessed using the PD scores from readers 1 and 2. The intermodality agreement between dual energy CT and SPECTCT was assessed using the scores from (I) readers 1 versus 3 and (II) readers 2 versus 3 . Note that because different readers used the two modalities, some inter-reader variability is present in the inter-modality agreement estimates.
When PD could not be graded with a particular modality (due to artifacts etc.), the affected observation was not included in the analysis.

Pearson's correlation coefficient was calculated as a measure of the linear correlation between percent enhancement measurements made with DECT and SPECT-CT. A bootstrap confidence interval for the correlation in measurements made across eight lung locations was computed as described previously.

\section{Results}

Figures 1 and 2 display the distribution of PD grades by reader over the 323 segments and 95 lobes, respectively.

The inter-reader agreement on DECT was fairly strong (Table 2). The two readers (1 and 2) agreed on the grade of PD in $85 \%(258 / 304)$ of segments (kappa $=0.86)$ and $84 \%(80 / 95)$ of lobes $(\mathrm{kappa}=0.82)$. The inter-modality agreement between DECT and SPECT-CT, however, was lower (Tables 3 and 4). Readers 1 and 3 agreed on the grading of PD in only $34 \%$ (103/304) of segments (kappa $=0.25)$ and $33 \%(31 / 94)$ of lobes (kappa $=0.22)$. Agreement between readers 2 and 3 was similar.

The correlation between the percent enhancement measurements with DECT and planar V/Q imaging ranged from 0.01 (in the right upper lung) to 0.45 (in the left upper lung). Across all locations, the correlation in percent enhancement measurements was 0.15 (95\% CI: 0.02-0.28). Figure 3 provides a visual.

The mean effective dose of DECT was $5.1 \pm 1.3 \mathrm{mSv}$ while that with SPECT-CT was $8.2 \pm 2.1 \mathrm{mSv}$.

\section{Discussion}

Our study demonstrates excellent inter-reader agreement in subjective grading of PD on DECT-PBV maps. However, inter-modality agreement of grading of perfusion on DECT and SPECT was modest. Automated quantification of perfusion on $\mathrm{PBV}$ also showed poor correlation with quantification perfusion by planar V/Q imaging.

Since the past decade, there has been increased understanding in the diagnosis and management of CTEPH. While the prognosis of $\mathrm{PH}$ is poor in general, CTEPH can be an exception. Early diagnosis of these patients is associated with good short- and long-term prognosis. While PTE is the definitive therapy of choice, in inoperable patients, balloon angioplasty (BPA) has emerged as a successful alternative (14). The diagnosis of CTEPH 
Table 2 Inter-reader agreement on dual energy CT (reader 1 vs. 2)

\begin{tabular}{|c|c|c|c|c|}
\hline Agreement type & \multicolumn{2}{|c|}{ Percent agreement } & \multicolumn{2}{|c|}{ Cohen's kappa } \\
\hline Segment-level & $85 \%(258 / 304)$ & $(81 \%, 89 \%)$ & 0.86 & $(0.81,0.90)$ \\
\hline Lobe-level & $84 \%(80 / 95)$ & $(77 \%, 91 \%)$ & 0.82 & $(0.72,0.90)$ \\
\hline Patient-level & $84 \%(16 / 19)$ & $(60 \%, 96 \%)$ & 0.74 & $(0.46,1.00)$ \\
\hline
\end{tabular}

Table 3 Inter-modality agreement (reader 1 on dual energy CT vs. reader 3 on SPECT-CT)

\begin{tabular}{lccccc}
\hline \multirow{2}{*}{ Agreement type } & \multicolumn{2}{c}{ Percent agreement } & \multicolumn{2}{c}{ Cohen's kappa } \\
\cline { 2 - 5 } & \multicolumn{1}{c}{ Estimate } & $95 \% \mathrm{Cl}$ & \multicolumn{2}{c}{ Estimate } & $(0.14,0.37)$ \\
Segment-level & $34 \%(103 / 304)$ & $(25 \%, 44 \%)$ & 0.25 & $(0.07,0.37)$ \\
Lobe-level & $33 \%(31 / 94)$ & $(21 \%, 46 \%)$ & 0.22 & $(-0.28,0.42)$ \\
Patient-level & $53 \%(10 / 19)$ & $(29 \%, 75 \%)$ & 0.07 & \\
\hline
\end{tabular}

Table 4 Inter-modality agreement (reader 2 on dual energy CT vs. reader 3 on SPECT-CT)

\begin{tabular}{|c|c|c|c|c|}
\hline Agreement type & \multicolumn{2}{|c|}{ Percent agreement } & \multicolumn{2}{|c|}{ Cohen's kappa } \\
\hline Segment-level & $36 \%(113 / 315)$ & $(27 \%, 45 \%)$ & 0.30 & $(0.19,0.42)$ \\
\hline Lobe-level & $34 \%(32 / 94)$ & $(24 \%, 45 \%)$ & 0.24 & $(0.10,0.37)$ \\
\hline Patient-level & $68 \%(13 / 19)$ & $(43 \%, 86 \%)$ & 0.25 & $(-0.16,0.66)$ \\
\hline
\end{tabular}

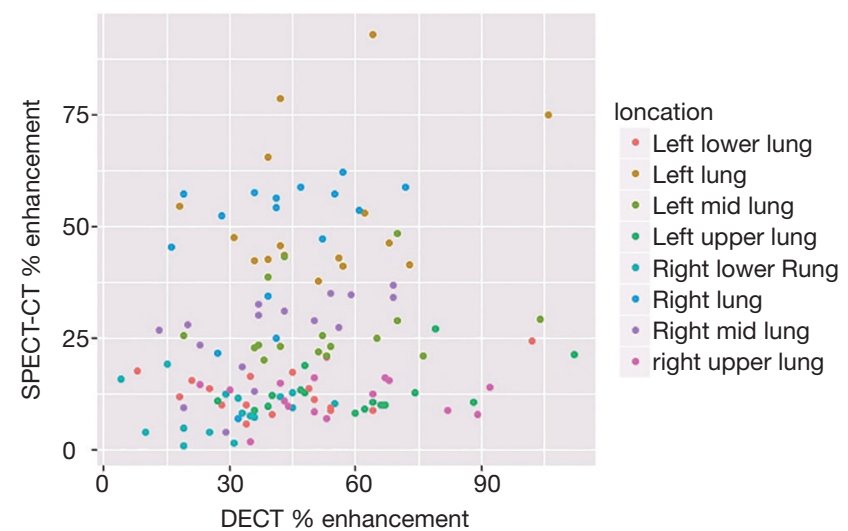

Figure 3 Correlation between DECT and SPECT-CT\% enhancement measurements across 8 locations in 16 patients.

can be challenging at times, and can be mimicked by group $1 \mathrm{PH}$ diseases such as idiopathic pulmonary arterial hypertension (IPAH). Beyond the diagnosis, deciding on intervention in these patients can be challenging at times, and often requires a multidisciplinary approach. Several factors need to be assessed, such as the distribution of disease, amount of thrombotic burden, hemodynamics such as PVR, and assessment of the lung macrocirculation and microcirculation. The distribution and amount of obstructive disease burden is very well assessed with CTPA. Assessment of PVR requires RHC, although noninvasive markers such as MRI based indices and PBV maps have been evaluated as predictors of PVR $(15,16)$.

Prior to intervention, it is also necessary to assess the lung segmental perfusion, the amount of perfusion abnormalities corresponding to the thrombotic burden, and the type of PD. For example, if there is a large amount of thrombotic burden in a lobar branch with corresponding wedge shaped perfusion abnormality in the same lobe, then it can be safely assumed that intervention in that area is acceptable. Traditionally, planar V/Q scanning has been used to assess the segmental and lobar perfusion and also to quantify the lung perfusion. Now, newer modalities such as DECT allow mapping of $\mathrm{PD}$ as well as automated quantification of blood 

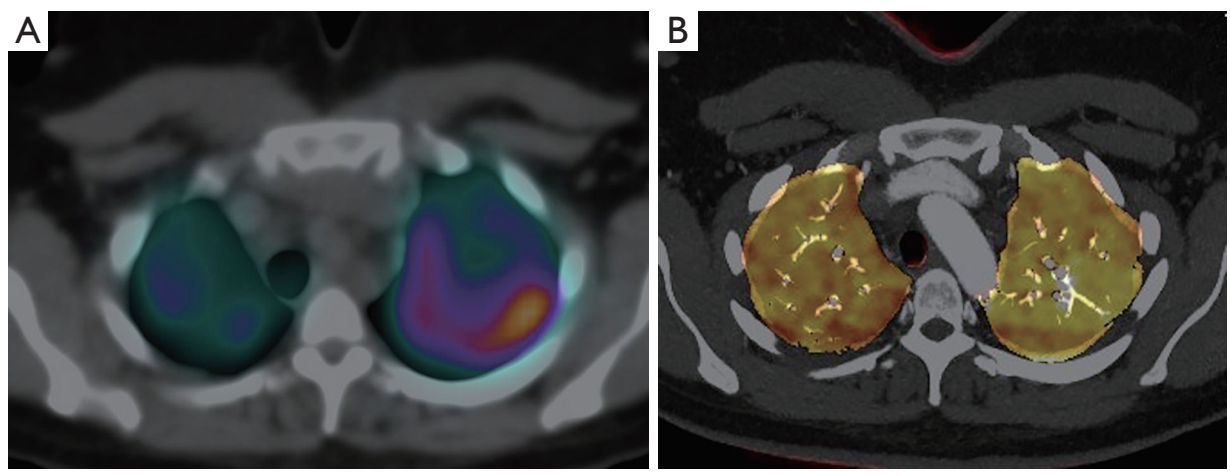

Figure 4 Representative example of discrepancy in the subjective assessment of perfusion on DECT and SPECT-CT V/Q scanning. (A) Fused SPECT-CT V/Q scan image in the upper lungs shows poor perfusion in the right upper lobe; (B) corresponding fused DECT-PBV image at the same level shows relatively symmetric and normal perfusion in both upper lobes.

volumes. However, since the methods used for calculation of perfusion are different with DECT versus V/Q scanning, caution needs to be exercised in interpreting the findings. Limited studies have shown modest to good correlation in gradation of PD. In a study on 40 patients, 20 out of 252 (7.9\%) segments were of non-diagnostic quality on iodine maps. There was a moderate agreement (raw agreement $=72 \%$, kappa value $=0.44$ (CI: $0.31-0.57)$ between DECT perfusion and V/Q scintigraphy (12). In another study on 51 patients, the agreement between $\mathrm{PBV}$ maps and planar V/Q scanning was good, with kappa value of 0.70 (13). A recent study by Giordano et al. on 31 patients reported concordant findings between DECT and planar V/Q in $100 \%$ of patients with peripheral CTEPH (17). In another study on canines using histopathology as a gold standard, DECT was found to have a higher accuracy than SPECT in detection of CTEPH (18). To address these conflicting issues, we sought to do a comprehensive comparative assessment of subjective and objective information obtained by DECT with both SPECT and planar V/Q imaging. Our results show a modest correlation between grading of PD on PBV maps and SPECT-CT V/Q with kappa values ranging from 0.25 to 0.30 (Figure 4). More strikingly, the correlation between quantification of perfusion using DECT and planar V/Q imaging was poor ranging from 0.01 (in the right upper lung) to 0.45 (in the left upper lung). Possible explanations for this poor correlation include method of perfusion estimation in DECT. PBV maps display the amount of iodine in a voxel and in actuality provide an estimate of relative perfusion, calculated in comparison to the enhancement of main pulmonary artery or aorta (in all of our studies, a manual region of interest was drawn on the main pulmonary artery to estimate quantification). Another potential explanation includes the potential of gravity induced and patient position induced preferential distribution of the tracer used in planar V/Q imaging to the dependent mid to lower lungs.

The indirect approach of identification of mismatched PD for diagnosis can work adequately in patients with suspected acute PE. However, in CTEPH, imaging is required to diagnose as well as assess the surgical candidacy of patient. In that respect, direct visualization of the thrombus distribution and burden is crucial. Hence, the combined usage of V/Q scanning and CT adds to the effective radiation dose burden to the patient. By allowing direct assessment of the thrombus burden and perfusion, DECT can allow to effectively cut down on patient radiation. Some concerns have been raised that DECT of chest might add to the radiation dose over single-source CT (19), although other studies have refuted this (20). In our study, the mean effective dose of DECT was $5.1 \pm 1.3 \mathrm{mSv}$ that is consistent to observations on prior studies (19).

Our study had several limitations. Firstly, the study population was small. This may be expected given that CTEPH is a fairly uncommon disease. Also, often patients are referred to our institution with prior imaging, which removes the clinical need for DECT and SPECT imaging. Ideally, a multi-institutional study might be of value to increase the number of patients evaluated. Also, some of the lung segments could not be assessed due to artifacts. Streak artifacts are a common occurrence with DECT, although the incidence has decreased with newer $2^{\text {nd }}$ and $3^{\text {rd }}$ generation scanners (17). In our studies, $5.5 \%$ of the 
lung segments were rendered nondiagnostic, which is similar to prior studies that have reported percentages of nondiagnostic segments in the range of $3.6 \%$ to $8.3 \%$ $(12,13,21)$. Another limitation of the study was that we compared only the perfusion data from two different imaging modalities. We did not take into account the obstructive disease burden seen on CTPA for our analysis. However, we wanted to specifically evaluate the interobserver agreement of PBV maps in grading PD and also compare perfusion information obtained using two novel imaging tools. One of the postulated explanations for the differences in perfusion information might be related to contrast bolus timing. In CTEPH patients, there is often a compensatory response with supply from bronchial artery collaterals. In one study, it has been shown that chronic PE segments might show improved perfusion on delayed scan, attributed to supply from collateral vessels (22). In our study, we just used the early phase CTPA protocol (timed to main pulmonary artery), which we acknowledge as a limitation.

\section{Conclusions}

In conclusion, DECT is a novel tool that allows assessment of anatomic and functional information regarding pulmonary thromboembolic disease in a single test. Interobserver agreement in subjective grading of PBV maps is excellent. However, caution needs to be exercised in interpreting the perfusion maps in comparison to SPECTCT V/Q imaging. Also, automated quantification values of PBV maps correlate poorly with established tools like planar V/Q imaging and caution needs to be exercised in use of these findings in clinical decision making. Larger, potentially multi-institutional studies may provide increased insight to the strengths and potential clinical applications for this emerging imaging technique.

\section{Acknowledgements}

None.

\section{Footnote}

Conflicts of Interest: The authors have no conflicts of interest to declare.

Ethical Statement: This retrospective study was performed with local institutional review board approval and a waiver of individual informed consent.

\section{References}

1. Lang IM, Pesavento R, Bonderman D, et al. Risk factors and basic mechanisms of chronic thromboembolic pulmonary hypertension: a current understanding. Eur Respir J 2013;41:462-8.

2. Galiè N, Hoeper MM, Humbert M, et al. Guidelines for the diagnosis and treatment of pulmonary hypertension: the task force for the diagnosis and treatment of pulmonary hypertension of the European Society of Cardiology (ESC) and the European Respiratory Society (ERS), endorsed by the International Society of Heart and Lung Transplantation (ISHLT). Eur Heart J 2009;30:2493-537.

3. Riedel M, Stanek V, Widimsky J, et al. Long-term followup of patients with pulmonary thromboembolism: late prognosis and evolution of hemodynamic and respiratory data. Chest 1982;81:151-8.

4. Fedullo P, Kerr KM, Kim NH, et al. Chronic thromboembolic pulmonary hypertension. Am J Respir Crit Care Med 2011;183:1605-13.

5. Corsico AG, D'Armini AM, Cerveri I, et al. Long-term outcome after pulmonary endarterectomy. Am J Respir Crit Care Med 2008;178:419-24.

6. Kim NH, Delcroix M, Jenkins DP, et al. Chronic thromboembolic pulmonary hypertension. J Am Coll Cardiol 2013;62:D92-9.

7. Tunariu N, Gibbs SJ, Win Z, et al. Ventilation-perfusion scintigraphy is more sensitive than multidetector CTPA in detecting chronic thromboembolic pulmonary disease as a treatable cause of pulmonary hypertension. J Nucl Med 2007;48:680-4.

8. Herald P, Roach P, Schembri GP. Does the addition of low dose CT improve diagnostic accuracy of V/Q SPECT scintigraphy?. J Nucl Med 2008;49:91P.

9. McCollough CH, Leng S, Yu L, et al. Dual- and multienergy CT: Principles, technical approaches, and clinical applications, Radiology 2015;276:637-53.

10. Fuld MK, Halaweish AF, Haynes SE, et al. Pulmonary perfused blood volume with dual-energy CT as surrogate for pulmonary perfusion assessed with dynamic multidetector CT. Radiology 2013;267:747-56.

11. Meinel FG, Graef A, Thierfelder KM, et al. Automated quantification of pulmonary perfused blood volume by dual-energy CTPA in chronic thromboembolic pulmonary hypertension. Rofo 2014;186:151-6.

12. Dournes G, Verdier D, Montaudon M, et al. Dual-energy 
CT perfusion and angiography in chronic thromboembolic pulmonary hypertension: diagnostic accuracy and concordance with radionuclide scintigraphy. Eur Radiol 2014;24:42-51.

13. Nakazawa T, Watanabe Y, Hori Y, et al. Lung perfused blood volume images with dual-energy computed tomography for chronic thromboembolic pulmonary hypertension: correlation to scintigraphy with singlephoton emission computed tomography. J Comput Assist Tomogr 2011;35:590-5.

14. Ogo T. Balloon pulmonary angioplasty for inoperable chronic thromboembolic pulmonary hypertension. Curr Opin Pulm Med 2015;21:425-31.

15. Hoey ET, Mirsadraee S, Pepke-Zaba J, et al. Dual-energy CT angiography for assessment of regional pulmonary perfusion in patients with chronic thromboembolic pulmonary hypertension: initial experience. AJR Am J Roentgenol 2011;196:524-32.

16. Swift AJ, Rajaram S, Hurdman J, et al. Noninvasive estimation of PA pressure, flow, and resistance with CMR imaging: derivation and prospective validation study from the ASPIRE registry. JACC Cardiovasc Imaging 2013;6:1036-47.

17. Giordano J, Khung S, Duhamel A, et al. Lung perfusion

Cite this article as: Renapurkar RD, Bolen MA, Shrikanthan S, Bullen J, Bullen W, Primak A, Heresi GA. Comparative assessment of qualitative and quantitative perfusion with dualenergy CT and planar and SPECT-CT V/Q scanning in patients with chronic thromboembolic pulmonary hypertension. Cardiovasc Diagn Ther 2018;8(4):414-422. doi: 10.21037/ cdt.2018.05.07 characteristics in pulmonary arterial hypertension (PAH) and peripheral forms of chronic thromboembolic pulmonary hypertension (pCTEPH): Dual-energy CT experience in 31 patients. Eur Radiol 2017;27:1631-9.

18. Tang CX, Yang GF, Schoepf UJ, et al. Chronic thromboembolic pulmonary hypertension: Comparison of dual-energy computed tomography and single photon emission computed tomography in canines. Eur J Radiol 2016;85:498-506.

19. de Broucker T, Pontana F, Santangelo T, et al. Singleand dual-source chest CT protocols: Levels of radiation dose in routine clinical practice. Diagn Interv Imaging 2012;93:852-8.

20. Schenzle JC, Sommer WH, Neumaier K, et al. Dual energy CT of the chest: how about the dose? Invest Radiol 2010;45:347-53.

21. Fink C, Johnson TR, Michaely HJ, et al. Dual-energy CT angiography of the lung in patients with suspected pulmonary embolism: initial results. Rofo 2008;180:879-83.

22. Hong YJ, Kim JY, Choe KO, et al. Different perfusion pattern between acute and chronic pulmonary thromboembolism: evaluation with two-phase dual-energy perfusion CT. AJR Am J Roentgenol 2013;200:812-7. 